\title{
Ocular Sebaceous Carcinoma
}

National Cancer Institute

\section{Source}

National Cancer Institute. Ocular Sebaceous Carcinoma. NCI Thesaurus. Code C43340.

The most common variant of sebaceous carcinoma that arises from the meibomian glands or sebaceous glands of the eyelid. 